\title{
Resiliencia de un pajonal de Panicum prionitis Nees bajo distintas alternativas de manejo
}

\author{
Ernesto S. Massa ${ }^{1, \otimes}$; Luis J. OAKLeY ${ }^{2}$; Darién E. Prado ${ }^{2,3} \&$ Susana R. Feldman ${ }^{2,3,4}$ \\ ${ }^{1}$ Instituto Nacional de Tecnología Agropecuaria, EEA Paraná, Oro Verde, Entre Ríos, Argentina. ${ }^{2}$ Facultad de Ciencias \\ Agrarias, Universidad Nacional de Rosario. Zavalla, Santa Fe, Argentina. ${ }^{3}$ Instituto de Investigaciones en Ciencias Agrarias de \\ Rosario (IICAR, CONICET-UNR). ${ }^{4}$ Consejo de Investigaciones de la Universidad Nacional de Rosario (CIUNR-UNR)
}

\begin{abstract}
Resumen. Los "pajonales" de Panicum prionitis ("paja de techar") son una de las comunidades de mayor extensión en las islas y el valle de inundación del río Paraná. Debido a sus tasas de crecimiento elevadas y a su baja calidad forrajera, tradicionalmente se los maneja con fuego para poder aprovecharlos en la actividad ganadera. El objetivo de este trabajo fue comparar los efectos de la quema y del corte del pajonal de P. prionitis sobre la vegetación (abundancia-cobertura, riqueza, equitatividad y diversidad). Para ello se condujo un experimento con distintos tratamientos: quema, corte (simulando una labor de desmalezado mecánico) y testigo sin disturbar, y se realizaron censos de vegetación durante dos años post-disturbio. P. prionitis mantuvo su condición de especie dominante, independientemente del tratamiento y a lo largo de todo el experimento. Aunque los disturbios alteraron la composición específica, aumentando la riqueza, la equitatividad y la diversidad con respecto al testigo, las diferencias desaparecieron luego de un año de haber aplicado los tratamientos. El corte tuvo el mismo efecto que el fuego, lo cual evidencia una gran resiliencia por parte de la comunidad en estudio.
\end{abstract}

[Palabras clave: disturbios, fuego, pastizales naturales, regeneración]

\begin{abstract}
Aвstract. Resilience of a Panicum prionitis Nees tall grassland under different management alternatives. Panicum prionitis ("paja de techar") tall grasslands are one of the most extensive vegetation types in the islands of the Parana River valley. These communities are traditionally burned in order to improve its quality forage and animal consumption. Our objective was to analyze the effect of different disturbances along two years: prescribed fire, mowing and control using multivariate analyses on vegetation variables (abundance-cover, richness, evenness and diversity). P. prionitis remained as dominant species regardless of treatment and throughout the experiment. Even though disturbances affected species composition, no significant effect was detected on richness, evenness, and diversity. Mowing and the use of prescribed fire showed the same effect on this highly resilient community.
\end{abstract}

[Keywords: disturbance, fire, rangelands, regeneration]

\section{INTRODUCCIÓN}

La vegetación del valle de inundación del Río Paraná pertenece a la Provincia Paranaense en el Dominio Amazónico (Cabrera 1971) y está influenciada por las crecientes anuales de los ríos Paraná y Paraguay (Franceschi and Lewis 1979). El área está cubierta por bosques de albardón o insulares (Franceschi et al. 1985), similares a los bosques marginales de la Selva Paranaense, y por bosques de sauces y alisos (géneros Salix y Tessaria, respectivamente) en los bancos de arena. Además, hay sabanas de Acacia caven (espinillares) (Franceschi et al. 1985), con una fisonomía dominante de bosque bajo y abierto, que alternan con abras mayormente cubiertas por pajonales altos y extensos. En estas abras, la gramínea Panicum prionitis Nees ("paja de techar", "paja de la isla") ejerce una dominancia fuerte y ocupa suelos inundables próximos a los grandes

Editora asociada: María Semmartin

massa.ernesto@inta.gob.ar cursos de agua (Franceschi and Prado 1989). El nombre de esta especie sufrió una modificación reciente y pasó a denominarse Coleataenia prionitis (Nees) Soreng (Soreng 2010). Sin embargo, a los fines prácticos de este trabajo se mantendrá el uso del nombre tradicional, ampliamente citado en la literatura.

Recientemente, la Secretaría de Ambiente de Entre Ríos (2016) caracterizó la cobertura del suelo para todo el Delta del Paraná. Se estimó que los pajonales y pastizales ocupan $\sim 570000$ ha (29\% del área). Los pajonales de P. prionitis se caracterizan por poseer dos estratos: uno alto dominado por matas de dicha especie y otro de bajo porte ubicado en el espacio entre matas. La intermata suele presentar especies herbáceas de los géneros Paspalum, Phalaris, Polypogon,Cyperus,Eleocharis, Rhynchospora, Scirpus, Eryngium, Solanum, Eupatorium,

Recibido: 17 de noviembre de 2015

Aceptado: 28 de septiembre de 2016 
Vernonia, Senecioy arbustivas de géneros como Mimosa y Sesbania (Ragonese 1967; Franceschi et al. 1985). Estos pajonales suelen tener matas mayores a $1.5 \mathrm{~m}$ de altura, y si su densidad es muy alta tienden a ser monoespecíficos (Sabattini et al. 1999).

En islas del tramo medio del río Paraná, Marchetti y Aceñolaza (2005) encontraron espacios cubiertos por estos pajonales y destacaron la presencia de dos estratos de vegetación. El superior alcanza hasta $3 \mathrm{~m}$ de altura, 70\% de cobertura, y está formado por "paja de techar" (P. prionitis), "carpinchera" (Mimosa pigra) y "café de la isla" (Sesbania virgata). En cambio, el estrato inferior es de $50 \mathrm{~cm}$ de altura y 95\% de cobertura, y posee mayor riqueza específica. Está formado por "gramilla" (Cynodon dactylon), "pasto horqueta" (Paspalum sp.), "cola de zorro" (Setaria parviflora), Paspalum simplex, "verdolaga"(Ludwigiapeploides),"paragüita" (Cyperusentrerianus)y Carexbonariensis, entre otras especies.

Los pajonales de $P$. prionitis sufren diferentes tipos de disturbios, tanto naturales como de origen antrópico. Los más frecuentes son el fuego, el pastoreo y las inundaciones periódicas. Todos ellos tienen implicancias sobre la estructura y la composición florística por un determinado tiempo, que depende de la intensidad del disturbio. El fuego es un componente natural de muchos ecosistemas de la Argentina y del mundo (Feldman and Lewis 2005; Felderhof and Gilyson 2006) y como parte de la dinámica de pastizales, pajonales y sabanas, provoca el rejuvenecimiento de estas comunidades, con un efecto positivo en aquellos ecosistemas explotados para la ganadería (Heringer and Jacques 2001). No obstante, Jacques (2003) advierte que al menos para los campos de altura del sur del Brasil, que presentan un nivel de endemismos elevado (Iganci et al. 2011), la quema rutinaria debería evitarse por sus efectos negativos sobre parámetros edáficos y, en algunos casos, forrajeros. En cambio, el mantenimiento de la diversidad y la fisonomía de los "campos" del sur del Brasil (Fidelis et al. 2010) está ligado a los disturbios provocados por la recurrencia del fuego y el pastoreo. Marchetti y Aceñolaza (2011) establecieron que el fuego favorece la persistencia de $P$. prionitis y preserva la riqueza y la diversidad de sus comunidades. Aunque no trabajaron específicamente con comunidades de $P$. prionitis, Salvia et al. (2012) concluyeron que un año después del fuego, las comunidades de los valles de inundación del delta del Paraná mostraban signos de recuperación en función directa a la severidad del mismo; esto favorecía la emergencia de especies intersticiales de las comunidades. Por su parte, Bissio (2014) determinó que luego de una quema, los rebrotes de las especies de pajonales (i.e., $P$. prionitis y otras gramíneas $\mathrm{C}_{4}$ del norte de la provincia de Santa $\mathrm{Fe}$ ) tendrían mejor aptitud forrajera que los pastos maduros. Con este criterio, los pajonales de $P$. prionitis se queman periódicamente como parte de su manejo (Feldman et al. 2008a). Sin embargo, desde el punto de vista del ambiente el uso del fuego resulta una práctica objetable dado que libera $\mathrm{CO}_{2}$ a la atmósfera. Por esta razón debería evitarse para atenuar el aumento de la concentración de gases de efecto invernadero (Figueroa Clemente and Redondo Gómez 2007).

En la búsqueda de prácticas alternativas a la quema de pajonales, postulamos que el corte podría tener un efecto agronómico semejante al del fuego, sin las emisiones de gases de efecto invernadero. El objetivo de este trabajo fue comparar los efectos de la quema y del corte del pajonal de $P$. prionitis sobre la estructura y composición florística de la comunidad.

\section{Materiales y Métodos}

El experimento se realizó en un pajonal del valle de inundación del río Paraná, dominado por $P$. prionitis. El pajonal estaba ubicado 40 $\mathrm{km}$ al sur de la ciudad de Alejandra, Santa Fe, Argentina $\left(29^{\circ} 9^{\prime} \mathrm{S}, 5^{\circ} 9^{\prime} \mathrm{O}\right)$. El suelo corresponde al orden Alfisol, Gran Grupo Natracualf típico (Soil Taxonomy 1999), familia franco fina, mixta, fuertemente alcalina y térmica, imperfectamente drenados a muy pobremente drenados, alcalinos en el subsuelo (Mosconi et al. 1981). El clima de la región es sub-húmedo-húmedo, mesotermal con una precipitación anual de $1250 \mathrm{~mm}$, concentrada en más del $80 \%$ en los meses de octubre y abril (promedio del período 1960-2012; EEA INTA Reconquista 2013).

El experimento comenzó en la primavera de 2011 (10 de octubre), siguiendo las prácticas frecuentes de los productores, quienes queman desde el comienzo de esa estación. Los tratamientos realizados fueron: 1) quema controlada (Q), 2) corte a una altura de $20 \mathrm{~cm}$ (simulando la labor de una desmalezadora rotativa) utilizando una moto-guadaña Hecho SRM, sin remover la vegetación cortada de la parcela (C), y 3) testigo sin disturbio (T). El 
diseño experimental utilizado fue en bloques completamente aleatorizados $(n=6)$, ubicados según la pendiente, totalizando 18 parcelas de $10 \times 10 \mathrm{~m}$. El predio experimental estuvo abierto a la fauna silvestre y con exclusión completa del pastoreo bovino desde 3 meses antes de iniciado el experimento.

El fuego se manejó con la técnica "en retroceso", recomendable cuando hay gran cantidad de biomasa combustible acumulada, ya que consume lentamente el oxígeno y permite un control mejor del frente de avance (Rodrigues et al. 2002). Cada parcela estuvo delimitada por cortafuegos de $1 \mathrm{~m}$ de ancho para evitar la propagación descontrolada del fuego. En las parcelas Q se midió la temperatura del suelo a tres profundidades: 1 ; 3 y $5 \mathrm{~cm}$, inmediatamente después de apagado el fuego (t0), a los 10 minutos (t10) y a los 30 minutos (t30). Para ello, se usó un termómetro digital Luft con rango de medición -50 a 150 ${ }^{\circ} \mathrm{C}\left( \pm 1{ }^{\circ} \mathrm{C}\right)$. Al inicio del experimento, la temperatura del aire se mantuvo entre 19.7 y $24^{\circ} \mathrm{C}$, con $53-68 \%$ HR y vientos $4-11 \mathrm{~km} / \mathrm{h}$ desde los cuadrantes S-SE-E. Las temperaturas del suelo durante la quema, en superficie y en profundidad, si bien tuvieron valores superiores a los de la temperatura del aire, nunca estuvieron por encima de valores que puedan llegar a afectar a los procesos biológicos, con mediciones entre 18 y $30^{\circ} \mathrm{C}$.

Previo a los tratamientos se había censado la vegetación del área experimental en parcelas de $4 \times 4 \mathrm{~m}$ ubicadas al azar $(\mathrm{n}=18)$. En ellas se registró la abundancia-cobertura (sensu Braun-Blanquet 1979) por especie, a las cuales se les asignó una clase de constancia en función del porcentaje del total de censos en los que estaban presentes (I: 1-20\%; II: 20.1-40\%; III: 40.1-60\%; IV: 60.1-80\% y V: 80.1$100 \%$ de presencia de esa especie en el total de parcelas censadas de ese tratamiento).

El 30 de diciembre de 2011 y el 1 de noviembre de 2012 (verano) se realizaron censos de vegetación en los que se registró la abundancia-cobertura (Braun-Blanquet 1979) por especie, en parcelas de $4 \times 4 \mathrm{~m}$ dentro de cada parcela experimental, y se asignó a cada especie una clase de constancia. Las fechas se establecieron con el objeto de encontrar a la mayor parte de las especies en estado reproductivo, lo cual facilita su determinación. Para la elaboración de las listas florísticas se siguió la nomenclatura de Pensiero et al. (2006). Se recopilaron antecedentes sobre las características de las especies presentes (tipo de metabolismo fotosintético $\left(\mathrm{C}_{3} \mathrm{O}\right.$
$\mathrm{C}_{4}$ ), tipo de reproducción (sexual o sexual y clonal), ciclo de vida (anual o perenne) y hábito de crecimiento (cespitosa, erecta, estolonífera, palustre, postrada o rastrera). Con la información de abundancia-cobertura recolectada en las dos fechas se confeccionaron sendas matrices de parcelas por especies, analizadas con métodos multivariados utilizando PC Ord (McCune and Mefford 1999), reemplazando los datos de abundanciacobertura de Braun-Blanquet por la escala de van der Maarel (1979). Se realizaron: 1) análisis de correspondencia, 2) análisis de especies indicadoras para establecer la existencia de especies que explicaran diferencias ante los disturbios (Peck 2010), 3) un procedimiento de permutación de respuestas múltiples (MRPP) usando la distancia euclídea y el cociente $n / \sum n$ para ponderar los grupos, y 4) la prueba de Friedman (Balzarini et al. 2008) para comparar los valores de diversidad (índice de Shannon: $\mathrm{H}=\Sigma \mathrm{p}_{\mathrm{i}}{ }^{*} \log \mathrm{p}_{\mathrm{i}}$ ) (Shannon 1948), riqueza (S, número de especies) y equitatividad $(\mathrm{E}=\mathrm{h} /$ $\ln [S]$ ) (Pielou 1969), para cada tratamiento y fecha de muestreo, usando Infostat (Balzarini et al. 2008).

\section{Resultados}

Se registró un total de 64 especies, con predominio de formas de vida perennes y escasas especies de buena o muy buena aptitud forrajera (ver Material suplementario) (Rossi et al. 2004; Luisoni 2006; Bissio 2014). La mayoría de ellas (46) correspondieron a la vía fotosintética $C_{3}$ y las 18 restantes a la $\mathrm{C}_{4}$. De estas últimas, 12 especies son Poaceae entre las que se destacan por su constancia y abundancia: P. prionitis, C. dactylon, Paspalum plicatulum, Chascolytrum uniolae y Setaria parviflora, todas nativas excepto $C$. dactylon. LasPoaceaeC $\mathrm{C}_{3}$ registradasfueron Chascolytrum uniolae, C. subaristatum, Phalaris angusta, Bromus catharticus, Deyeuxia viridiflavescens, Leersia hexandra y Poa annua, de las cuales esta última es la única exótica. Otra familia con alto número de especies $\mathrm{C}_{3}$ fue Asteraceae, con 12 representantes, de baja presencia. Las cuatro especies nativas de Fabaceae son $\mathrm{C}_{3}$ y de buena aptitud forrajera: Desmodium incanum, Dolichopsis paraguariensis, Mimosa strigillosa y Desmanthus virgatus. Todas estas son de porte semi-erectas o rastreras, se ubican en la intermata y, en el caso de $D$. paraguariensis, es postrada y con la capacidad de entrelazarse dentro de la mata de $P$. prionitis.

El censo previo a la aplicación de los tratamientos presentó 37 especies: 22 
latifoliadas y 15 graminiformes (Tabla 1). La cobertura fue $90-100 \%$, y $P$. prionitis fue la especie dominante. Las especies más constantes, con valores de cobertura menores, fueron: C. dactylon, Echinodorus grandiflorus, Oxalis corniculata y Paspalum plicatulum. Las Cyperaceae censadas son nativas y perennes: Cyperus entrerianus, Fimbristylis squarrosa, RhynchosporacorymbosayEleocharismontana, mientras que las Fabaceae, Desmodium incanum, Dolichopsisparaguariensisy Mimosa strigillosa, son nativas y perennes, con baja constancia inicial. Otra familia botánica con un número importante de especies fue la Asteraceae, pero con baja constancia, excepto Mikania cordifolia (Tabla 1). Panicum prionitis fue siempre la especie dominante, mostrando la máxima constancia independientemente del tratamiento y en ambas fechas de censado.

El censo de diciembre de 2011 denotó el efecto del corte o la quema sobre el número total de especies con respecto a las parcelas control, con un incremento relativo de las especies graminiformes. En las parcelas control, $60 \%$ de las especies graminiformes fueron Poaceae, en su mayoría perennes, exceptuando las anuales Setariaparvifloray Bromuscatharticus.Laúnica Fabaceae registrada fue Mimosa strigillosa. Esa contribución llegó a 69\% de Poaceae en las parcelas control, con especies de buena aptitud forrajera pero baja constancia: Paspalum plicatulum, Bothriochloa longipaniculata y Paspalum urvillei con la máxima constancia, después de $P$. prionitis; Desmanthus virgatus fue la única Fabaceae censada. Las parcelas quemadas presentaron la mayor cantidad de graminiformes $y$, a su vez, mostraron la mayor proporción de Poaceae. Estas parcelas incluyeron de manera exclusiva especies como Hemarthriaaltissimay Deyeuxiaviridiflavescens, ambas perennes y de buena aptitud forrajera. Mimosa strigillosa persistió pero con baja cobertura. Chascolytrum subaristatum, especie invernal de muy buena aptitud forrajera, tuvo baja constancia en las parcelas cortadas y en las quemadas. Las Cyperaceae no alteraron su abundancia y cobertura en función de los tratamientos. $P$. prionitis se mantuvo como dominante tanto por constancia como por cobertura: cerca del $100 \%$ en las parcelas control, más del $50 \%$ en las cortadas y entre $40 \%$ y $50 \%$ en las quemadas.

Los primeros dos ejes del análisis de correspondencia absorbieron un alto porcentaje de la varianza (14.54 y $12.79 \%$, respectivamente) (Figura 1a). Las parcelas testigo se ubicaron hacia el lado negativo del eje 2, con pocas especies exclusivas: Mikania cordifolia, Eupatorium macrocephalum, E. lanigerum, E. arnottianum, Justicia laevilinguis, Sida rhombifolia, Bromuscatharticusy Acmella decumbens. Las parcelas disturbadas se ubicaron en todos los cuadrantes. La mayoría de las parcelas quemadas se ubicaron a lo largo de valores positivos del primer eje, con los mayores valores de cobertura de Cynodon dactylon y no se encontraron algunas de las especies registradas inicialmente (Echinodorus grandifforus, Alternantheraphiloxeroides,Mikania cordifolia,Polygonumconvolvulus)ylasparcelas cortadas quedaron ubicadas a lo largo de todo el primer eje, sin un patrón evidente. En las parcelas cortadas se registró la presencia de Paspalum urvilleiy P. plicatulum. Bothriochloa longipaniculata se encontró sólo en este tratamiento, al igual que algunas latifoliadas como Oxalis corniculata, Chenopodium ambrosioides,Conyzabonariensisy Eupatorium macrocephalum.

El censo de noviembre de 2012 presentó, en las parcelas cortadas, especies perennes y nativas (exceptuando Echinochloa colona, Poa annua y Bromus catharticus, que son anuales) y de buena calidad forrajera. Las Cyperaceae mantuvieron e incluso aumentaron sensiblemente su cobertura, al igual que Alternantheraphiloxeroides.Estopudodeberse a la elevada cantidad de precipitación que se registró a principios de octubre de 2012. Las Fabaceae persistieron con baja cobertura en el pajonal, independientemente de los disturbios (Tabla 2).

Los dos primeros ejes del análisis de correspondencia absorbieron apenas 12.09\% y $11.48 \%$ de la varianza (Figura 1b). Las parcelas testigo se concentraron sobre los valores negativos del primer eje, con la permanencia de Alternanthera philoxeroides, Polygonum acuminatum, Cuphea glutinosa, Cyperus entrerianus, Fimbristylis squarrosa, Paspalum intermedium, $P$. urvillei, Phalaris angusta, Solanum glaucophyllum y Baccharis trimera (los dos últimos, arbustos sub-leñosos). Las parcelas disturbadas, si bien más dispersas en el plano, estuvieron alejadas de las testigo, probablemente por aumentos ligeros de las Asteraceae Xanthium spinosum, Eupatorium arnottianum, E. lanigerumy E. macrocephalum y de otras latifoliadas (i.e., Parietaria debilis, Phyla nodiflora) sin valor forrajero y valores bajos de constancia, y también de otras que sí tienen valor forrajero como Chascolytrum subaristatum,Paspalumintermedium,P.urvillei y Deyeuxia viridiflavescens. Los análisis de 
Tabla 1. Pajonal de P. prionitis: composición florística, constancia (en números romanos) y valor promedio de abundancia y cobertura (subíndice) en las parcelas T: testigo, C: corte y Q: quema, en las fechas de censado. Las referencias de constancia indican, I: 1-20\%; II: 20.1-40\%; III: 40.1-60\%; IV: $60.1-80 \%$ y V: $80.1-100 \%$ de presencia de esa especie en el total de parcelas censadas de ese tratamiento.

Table 1. P. prionitis tall grassland: floristic composition, constancy (Roman numerals) and average cover abundance values (Subscript) in control $(\mathrm{T})$, mowed $(\mathrm{C})$, and burnt $(\mathrm{Q})$ plots in the sampling dates.

\begin{tabular}{|c|c|c|c|c|c|c|c|}
\hline \multirow[b]{2}{*}{ Especies } & \multirow{2}{*}{$\begin{array}{l}\text { Situación } \\
\text { inicial }\end{array}$} & \multicolumn{3}{|c|}{$30 / 12 / 2011$} & \multicolumn{3}{|c|}{$1 / 12 / 12$} \\
\hline & & $\mathrm{T}$ & $\mathrm{C}$ & Q & $\mathrm{T}$ & $\mathrm{C}$ & Q \\
\hline Panicum prionitis & $\mathrm{V}_{48}$ & $\mathrm{~V}_{45}$ & $\mathrm{~V}_{30}$ & $\mathrm{~V}_{28}$ & $\mathrm{~V}_{5}$ & $\mathrm{~V}_{45}$ & $\mathrm{~V}_{37}$ \\
\hline Cynodon dactylon & III $_{07}^{4.8}$ & IIII $_{07}^{4.5}$ & $\mathrm{III}_{03}^{3.0}$ & $\mathrm{III}_{21}^{2.8}$ & $\mathrm{I}_{0.3}^{5}$ & $\mathrm{I}_{07}^{4.5}$ & $\mathrm{II}_{10}^{3.7}$ \\
\hline Echinodorus grandiflorus & III $_{0.4}^{0.7}$ & IIII $_{0.5}^{0.7}$ & $\mathrm{II}_{0.3}^{0.3}$ & & & & \\
\hline Oxalis corniculata & $\mathrm{III}_{0.5}^{0.4}$ & & $\mathrm{I}_{0.2}^{0.3}$ & $\mathrm{I}_{0.2}$ & $\mathrm{II}_{0.3}$ & $\mathrm{I}_{0.3}$ & $\mathrm{I}_{0.3}$ \\
\hline Paspalum plicatulum & III $_{0.4}^{0.5}$ & & III $_{0.5}^{0.2}$ & $\mathrm{III}_{1.0}^{0.2}$ & $\mathrm{I}_{0.2}^{0.3}$ & $\mathrm{II}_{1.0}^{0.3}$ & $\mathrm{II}_{1.3}^{0.3}$ \\
\hline Alternanthera philoxeroides & $\mathrm{II}_{0.2}^{0.4}$ & $\mathrm{I}_{0.2}$ & $\mathrm{I}_{0.3}^{0.5}$ & & III $_{0.7}^{0.2}$ & $\mathrm{IV}_{1.2}^{1.0}$ & III $_{0.7}^{1.3}$ \\
\hline Chascolytrum uniolae & $\mathrm{II}_{0.5}^{0.2}$ & & & & & II $_{0.3}^{1.2}$ & $\mathrm{I}_{0.2}^{0.7}$ \\
\hline Eleocharis montana & II ${ }_{02}^{0.5}$ & & & & & & \\
\hline Hyptis lappacea & $\mathrm{II}_{0.4}^{0.2}$ & $\mathrm{I}_{0.2}$ & $\mathrm{II}_{0.3}$ & $\mathrm{II}_{0.3}$ & $\mathrm{I}_{0.2}$ & $\mathrm{I}_{0.2}$ & $\mathrm{II}_{0.3}$ \\
\hline Mikania cordifolia & $\mathrm{II}_{0.4}^{0.4}$ & $\mathrm{I}_{0.2}^{0.2}$ & $\mathrm{I}_{0.2}^{0.3}$ & & & $\mathrm{I}_{0.2}^{0.2}$ & \\
\hline Polygonum acuminatum & $\mathrm{II}_{0.3}^{0.4}$ & $\mathrm{II}_{0.3}^{0.2}$ & $\mathrm{I}_{0.3}^{0.2}$ & $\mathrm{II}_{0.2}$ & $\mathrm{II}_{0.8}$ & $\mathrm{I}_{0.3}^{0.2}$ & $\mathrm{I}_{0.3}$ \\
\hline Polygonum convolvulus & $\mathrm{I}_{02}^{0.3}$ & & & $\mathrm{I}_{0.2}^{0.2}$ & & & \\
\hline Setaria parviflora & $\mathrm{II}_{0.3}^{0.2}$ & $\mathrm{I}_{03}$ & $\mathrm{I}_{0.5}$ & $\mathrm{III}_{0.2}^{0.2}$ & & & $\mathrm{I}_{0.5}$ \\
\hline Aspilia montevidensis & $\mathrm{I}_{0.1}^{0.3}$ & & & & & & \\
\hline Chascolytrum subaristatum & $\mathrm{I}_{0.1}^{0.1}$ & & $\mathrm{I}_{0.2}$ & $\mathrm{I}_{0.2}$ & & $\mathrm{I}_{0.5}$ & $\mathrm{I}_{0.2}$ \\
\hline Chenopodium ambrosioides & $\mathrm{I}_{0.2}^{0.1}$ & & III $_{0.5}^{0.2}$ & $\mathrm{II}_{0.3}^{0.2}$ & & & \\
\hline Conyza bonariensis & $\mathrm{I}_{0.1}^{0.2}$ & & $\mathrm{I}_{0.2}^{0.5}$ & & & & \\
\hline Cuphea glutinosa & $\mathrm{I}_{0.1}^{0.1}$ & $\mathrm{I}_{0.3}$ & & & $\mathrm{I}_{0.2}$ & & $\mathrm{I}_{0.2}$ \\
\hline Cyperus entrerianus & $\mathrm{I}_{0.3}^{0.1}$ & $\mathrm{I}_{0.2}^{0.3}$ & $\mathrm{I}_{02}$ & $\mathrm{III}_{07}$ & $\mathrm{I}_{02}^{0.2}$ & $\mathrm{II}_{03}$ & III \\
\hline Desmodium incanum & $\mathrm{I}_{0.2}^{0.3}$ & & $\mathrm{I}_{0.3}^{0.2}$ & & & $\mathrm{I}_{0.2}^{0.3}$ & \\
\hline Dolichopsis paraguariensis & $\mathrm{I}_{0.1}^{0.2}$ & & & & & & \\
\hline Eragrostis lugens & $\mathrm{I}_{0.1}^{0.1}$ & & & & & & \\
\hline Eryngium ebracteatum & $\mathrm{I}_{0.1}^{0.1}$ & & $\mathrm{I}_{0.2}$ & $\mathrm{II}_{0.3}$ & & $\mathrm{II}_{0.3}$ & $\mathrm{III}_{1.0}$ \\
\hline Fimbristylis squarrosa & $\mathrm{I}_{0.2}^{0.1}$ & $\mathrm{I}_{0.3}$ & & $\mathrm{II}_{0.7}^{0.3}$ & $\mathrm{I}_{0.3}$ & $\mathrm{I}_{0.2}^{0.3}$ & \\
\hline Acroceras zizanioides & $\mathrm{I}_{0.2}^{0.2}$ & & & & & & \\
\hline Gamochaeta coarctata & $\mathrm{I}_{0.1}^{0.2}$ & & & & & & \\
\hline Hydrocotyle bonariensis & $\mathrm{I}_{0.2}^{0.1}$ & & & & & III $_{0.5}$ & \\
\hline Ludwigia peploides & $\mathrm{I}_{0.2}^{0.2}$ & & & $\mathrm{I}_{02}$ & & & \\
\hline Mimosa strigillosa & $\mathrm{I}_{0.1}^{0.2}$ & $\mathrm{I}_{02}$ & & $\mathrm{I}_{0.2}^{0.2}$ & & $\mathrm{I}_{02}$ & III $_{07}$ \\
\hline Paspalum intermedium & $\mathrm{I}_{0.1}^{0.1}$ & $\mathrm{II}_{0.5}^{0.2}$ & IIII $_{0.3}$ & $\mathrm{II}_{1.2}^{0.2}$ & $\mathrm{I}_{0.3}$ & $\mathrm{I}_{0.5}^{0.2}$ & $\mathrm{I}_{1.2}^{0.7}$ \\
\hline Paspalum urvillei & $\mathrm{I}_{0.2}^{0.1}$ & & $\mathrm{IV}_{1.2}^{0.3}$ & $\mathrm{I}_{0.2}^{1.2}$ & $\mathrm{I}_{0.3}^{0.3}$ & III $_{1.2}^{0.5}$ & $\mathrm{IV}_{1.5}^{1.2}$ \\
\hline Phalaris angusta & $\mathrm{I}_{0.1}^{0.2}$ & & & & & $\mathrm{IV}_{0.8}^{1.2}$ & $\mathrm{IV}_{1.2}^{1.5}$ \\
\hline Pteris tremula & $\mathrm{I}_{0.2}^{0.1}$ & & & & $\mathrm{I}_{0.2}^{0.7}$ & & \\
\hline Rhynchospora corymbosa & $\mathrm{I}_{0.1}^{0.2}$ & & $\mathrm{I}_{0.2}$ & & $\mathrm{I}_{0.2}^{0.2}$ & $\mathrm{I}_{0.2}$ & $\mathrm{I}_{0.2}$ \\
\hline Solanum glaucophyllum & $\mathrm{I}_{0.1}^{0.1}$ & & & & $\mathrm{I}_{0.2}^{0.2}$ & $\mathrm{I}_{0.2}^{0.2}$ & \\
\hline Stellaria media & $\mathrm{I}_{0.1}^{0.1}$ & & & & & & \\
\hline Xanthium spinosum & $\mathrm{I}_{0.1}^{0.1}$ & & & $\mathrm{I}_{02}$ & & & $\mathrm{I}_{02}$ \\
\hline Acmella decumbens & & $\mathrm{I}_{0.2}$ & & & & & \\
\hline Baccharis trimera & & & & & $\mathrm{II}_{0.5}$ & $\mathrm{I}_{033}$ & \\
\hline Bothriochloa longipaniculata & & & $\mathrm{I}_{02}$ & & & $\mathrm{I}_{0.3}$ & $\mathrm{I}_{02}$ \\
\hline Bromus catharticus & & $\mathrm{I}_{0.2}$ & & & & $\mathrm{I}_{0.3}^{0.2}$ & \\
\hline Convolvulus arvensis & & & & & $\mathrm{I}_{0.2}$ & $\mathrm{I}_{0.2}^{0.3}$ & $\mathrm{I}_{0.2}$ \\
\hline Cyperus sp. & & & & $\mathrm{I}_{0.2}$ & $\mathrm{I}_{0.2}^{0.2}$ & $\mathrm{I}_{0.2}^{0.2}$ & \\
\hline Desmanthus virgatus & & & $\mathrm{I}_{0.2}$ & & & $\mathrm{I}_{0.3}^{0.2}$ & $\mathrm{I}_{03}$ \\
\hline Deyeuxia viridiflavescens & & & & $\mathrm{I}_{0.2}$ & $\mathrm{II}_{0.3}$ & $\mathrm{II}_{0.5}^{0.3}$ & $\mathrm{II}_{0.5}^{0.3}$ \\
\hline Echinochloa colona & & & & & & $\mathrm{I}_{0.2}^{0.3}$ & \\
\hline Eleocharis elegans & & $\mathrm{I}_{0.2}$ & $\mathrm{I}_{0.2}$ & & $\mathrm{I}_{0.2}$ & & \\
\hline Eupatorium arnottianum & & $\mathrm{I}_{0.2}^{0.2}$ & & & $\mathrm{II}_{07}^{0.2}$ & $\mathrm{I}_{03}$ & $\mathrm{I}_{03}$ \\
\hline Eupatorium lanigerum & & $\mathrm{I}_{0.2}^{0.2}$ & & & & $\mathrm{I}_{0.2}^{0.3}$ & $\mathrm{I}_{03}^{0.3}$ \\
\hline Eupatorium macrocephalum & & & $\mathrm{I}_{0.2}$ & & $\mathrm{I}_{0.5}$ & & $\mathrm{I}_{0.2}^{0.3}$ \\
\hline Leersia hexandra & & $\mathrm{I}_{0.2}$ & & $\mathrm{I}_{0.3}$ & & & \\
\hline Hemarthria altissima & & & & $\mathrm{I}_{0.2}^{0.3}$ & & & \\
\hline Justicia cf. laevilinguis & & $\mathrm{II}_{0.3}$ & $\mathrm{II}_{0.3}$ & & & & \\
\hline Lamium amplexicaule & & & & & & $\mathrm{II}_{0.3}$ & $\mathrm{I}_{0.2}$ \\
\hline Mikania periplocifolia & & $\mathrm{II}_{0.2}$ & III $_{0.2}$ & & & $\mathrm{I}_{0.2}^{0.3}$ & \\
\hline Parietaria debilis & & & & & & $\mathrm{I}_{0.3}^{0.2}$ & \\
\hline Phyla nodiflora & & & & & & & $\mathrm{I}_{02}$ \\
\hline Poа апnиа & & & & & $\mathrm{I}_{0.2}$ & $\mathrm{II}_{1.3}$ & $\mathrm{IV}_{1.5}$ \\
\hline Sacciolepis vilvoides & & $\mathrm{II}_{0.3}$ & $\mathrm{I}_{0.2}$ & $\mathrm{I}_{0.2}$ & & & \\
\hline Schoenoplectus californicus & & & & & & & $\mathrm{I}_{0.2}$ \\
\hline Sida rhombifolia & & & $\mathrm{I}_{0.2}$ & $\mathrm{I}_{0.2}$ & & & \\
\hline Vernonia glabrata var. angustifolia & & & & $\mathrm{I}_{0.3}^{0.2}$ & $\mathrm{I}_{02}$ & $\mathrm{II}_{03}$ & \\
\hline Nierembergia aristata & & & & & & $\mathrm{I}_{0.2}^{0.3}$ & $\mathrm{I}_{0.3}$ \\
\hline Sisyrinchium sp. & & & & & & $\mathrm{I}_{0.2}^{0.2}$ & $\mathrm{I}_{0.3}^{0.3}$ \\
\hline
\end{tabular}


Tabla 1. Continuación

Table 1. Continuation

\begin{tabular}{lccccccc}
\hline \multicolumn{1}{c}{ Especies } & Situación & \multicolumn{3}{c}{$30 / 12 / 2011$} & \multicolumn{3}{c}{$1 / 12 / 12$} \\
\hline total especies & inicial & $\mathrm{T}$ & $\mathrm{C}$ & $\mathrm{Q}$ & $\mathrm{T}$ & $\mathrm{C}$ & $\mathrm{Q}$ \\
№ graminiformes & 37 & 22 & 27 & 25 & 26 & 37 & 31 \\
\% graminiformes & 15 & 9 & 12 & 13 & 13 & 16 & 14 \\
№ latifoliadas & 40.5 & 45.5 & 44.4 & 52.0 & 50.0 & 43.2 & 45.2 \\
\% latifoliadas & 22 & 12 & 15 & 11 & 13 & 21 & 17 \\
\hline
\end{tabular}

Tabla 2. Comparación de los valores de diversidad $\left(\mathrm{H}^{\prime}\right)$ y sus componentes: riqueza (S) y equitatividad (E), entre parcelas testigo, cortadas y quemadas $(n=6$; dentro de cada fecha, valores seguidos por igual letra no difieren entre sí, Friedman, $P<0.05)$.

Table 2. Comparison among diversity values $\left(\mathrm{H}^{\prime}\right)$ and its components: richness (S) and evenness (E), among control, mowed, and burnt plots $(n=6$; for each census, values followed by the same letter are not statistically different, Friedman $P<0.05)$.

\begin{tabular}{lccc}
\hline & Testigo & $\begin{array}{c}\text { Corte } \\
30 / 12 / 2011\end{array}$ & Quema \\
\hline $\mathrm{H}^{\prime}$ & $1.67 \mathrm{a}$ & $2.5 \mathrm{a}$ & $1.83 \mathrm{a}$ \\
$\mathrm{S}$ & $10 \mathrm{a}$ & $15 \mathrm{a}$ & $11 \mathrm{a}$ \\
$\mathrm{E}$ & $1.17 \mathrm{~b}$ & $2.5 \mathrm{a}$ & $2.33 \mathrm{a}$ \\
& & $01 / 12 / 2012$ & \\
\hline $\mathrm{H}^{\prime}$ & $1.17 \mathrm{~b}$ & $2.17 \mathrm{a}$ & $2.67 \mathrm{a}$ \\
$\mathrm{S}$ & $7.5 \mathrm{~b}$ & $13.5 \mathrm{a}$ & $15 \mathrm{a}$ \\
$\mathrm{E}$ & $1.17 \mathrm{~b}$ & $2.08 \mathrm{a}$ & $2.75 \mathrm{a}$ \\
\hline
\end{tabular}

especies indicadoras no permitieron detectar que alguna especie aportara información sobre los tratamientos, en ninguno de los años analizados $(P<0.05)$.

A pesar de que en diciembre de 2011 la diversidad, la riqueza y la equitatividad de las parcelas cortadas fueron mayores que las de los restantes tratamientos, la prueba de permutación de respuestas múltiples no reveló diferencias estadísticamente significativas. No obstante, la prueba de Friedman mostró que la equitatividad en las parcelas testigo fue significativamente menor a la de las otras parcelas. Un año más tarde, la prueba de permutación de respuestas múltiples detectó diferencias entre tratamientos, probablemente debido a que las parcelas disturbadas tuvieron mayores valores de diversidad, riqueza y equitatividad con respecto a las testigo, sin diferir significativamente entre ellas (Tabla 2).

\section{DisCUSIÓN}

Los resultados de este estudio permitieron corroborar las evidencias de otros autores acerca de la ausencia de cambios drásticos en la composición florística del pajonal frente

a disturbios por quema y corte. Heringer y Jacques (2001) observaron cambios en la dinámica y la asociación de especies en comunidades adaptadas al fuego, con las gramíneas como el componente más tolerante, por el continuo crecimiento de meristemas intercalares y de los macollos protegidos por las vainas foliares. En un pajonal de Paspalumquadrifariumdelapampadeprimida (provincia de Buenos Aires), la cubierta vegetal se restableció a valores similares a los manchones del pajonal virgen en la primera temporada de crecimiento después del fuego (Laterra 2003). Coincidentemente, Peláez et al. (2003) sostienen que la ocurrencia de un único fuego no produce el reemplazo de especies ya que la comunidad vegetal se mantiene con leves variaciones en la abundancia de las principales gramíneas. Esto permite explicar por qué bajo nuestras condiciones, tanto a los pocos meses del disturbio como al final del experimento $P$. prionitis continuó siendo la especie más constante y con mayor abundancia y cobertura, y las demás especies del pajonal no desaparecieron masivamente. La estructura esencial de la comunidad se mantuvo a lo largo del tiempo y a pesar de los disturbios. De las 37 especies censadas originalmente, sólo ocho no volvieron a ser censadas. Si bien la dominante es una planta $\mathrm{C}_{4}$, en el resto de la comunidad predominan notoriamente las especies $C_{3^{\prime}}$ lo cual concuerda con el hecho de que no se trata de una comunidad que sufra un acentuado estrés hídrico, ya sea por déficit de precipitaciones o exceso de salinidad (Feldman et al. 2008b). De este modo, en este estudio ninguno de los dos tratamientos (corte y quema) provocó ni una acentuada ni una peligrosa pérdida de biodiversidad en la comunidad original, lo que revela su grado elevado de resiliencia y tolerancia a los disturbios.

La recuperación de la cobertura luego de los disturbios fue rápida, al igual que lo observado por Franceschi (2005) luego de una inundación catastrófica del río Paraná, donde en una primera etapa el sustrato fue ocupado por especies de ciclo corto y luego con rebrotes 

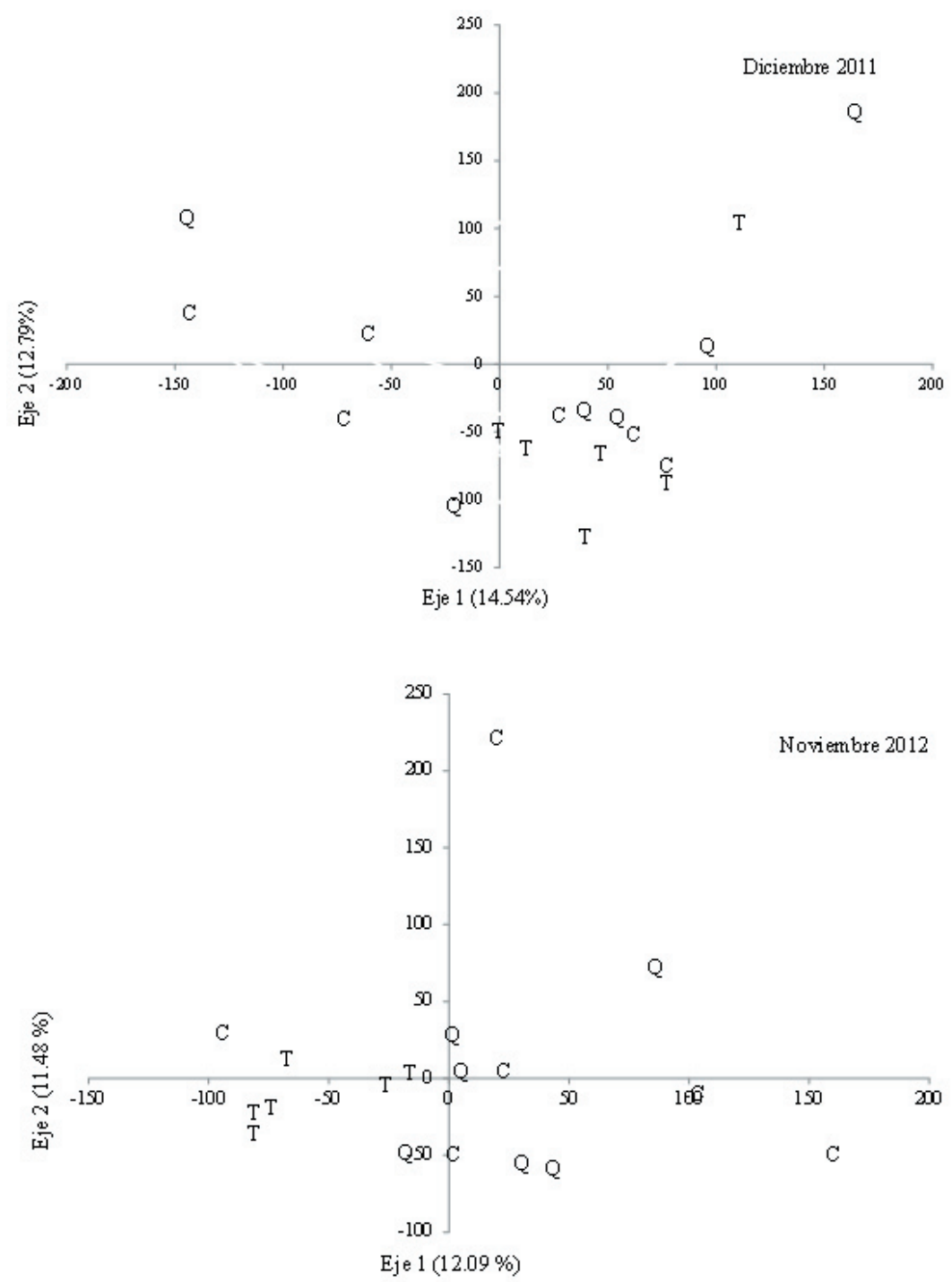

Figura 1. Diagrama de dispersión en el plano de los ejes 1 y 2 de las parcelas sometidas a distintos tratamientos: testigo $(\mathrm{T})$, cortadas $(\mathrm{C}) \mathrm{y}$ quemadas $(\mathrm{Q})$. Censos del 30 de diciembre de 2011 y del 1 de noviembre de 2012.

Figure 1. Scatter diagram with the first and second principal components of control $(\mathrm{T})$, mowed (C), and burnt $(\mathrm{Q})$ plots. Census December 30 2011 and November 1, 2012.

de las matas de $P$. prionitis sobrevivientes (Lewis et al. 1987; Franceschi 2005). En nuestro caso no ocurrió ese modelo sucesional: la vegetación se regeneró principalmente a partir de los rebrotes de las matas de $P$. prionitis. Las especies de la intermata post-disturbio fueron las mismas que ya estaban en la matriz del pajonal debido a que la magnitud del disturbio en nuestras parcelas fue inferior a la analizada por Franceschi (2005) y las especies pudieron retomar rápidamente el crecimiento postdisturbio o emerger del banco de semillas, que no se vio afectado.

La segunda especie en importancia por su valor de constancia y abundancia, Cynodon dactylon, es considerada una maleza muy agresiva en sistemas agrícolas, y aunque su calidad forrajera no es óptima, en este tipo de ambientes puede ser un recurso aceptable (Oakley 1999). Bajo nuestras condiciones experimentales, $C$. dactylon se vio afectada de forma negativa, pero dado que se trata de una planta estolonífera, estimamos que a mediano plazo recuperaría su espacio. Las otras Poaceae y graminiformes fueron poco afectadas por los disturbios o, en algunos casos, se establecieron una vez que se verificó una apertura en el canopeo. Las Fabaceae tampoco se vieron muy afectadas por los tratamientos experimentales, y si bien no aportan un gran volumen de forraje, su presencia probablemente sea significativa para la economía del nitrógeno.

Cynodon dactylon, Cyperus entrerianus y Paspalum intermedium aumentaron su abundancia-cobertura en las parcelas Q. Paspalum plicatulum tuvo aumentos de abundancia-cobertura bajo los dos tipos de disturbio, mientras que $P$. urvillei solo en el tratamiento de corte, similar a lo reportado por Jacques (2003). Eryngium ebracteatum no fue afectada por los disturbios, siguiendo el 
patrón encontrado por Fidelis et al. (2010), quienes observaron que especies del género Eryngium rebrotaron rápidamente una semana después de la quema, debido a que la roseta no se ve afectada por las llamas. En cambio, Alternantheraphiloxeroidestuvounatendencia diferente a la registrada por Salvia et al. (2012), en pajonales del bajo delta post-incendio. En nuestro caso estuvo desde el inicio y solo bajó la constancia a los 80 días de iniciado el experimento en las parcelas testigo y en las cortadas, aumentando al año en ambos tratamientos. Por el contrario, en las parcelas quemadas tardó en recuperarse hasta pasado un año.

Neiff (2001) sostiene que el fuego prescripto favorece el mantenimiento de la biodiversidad porque evita la acumulación de combustible en el suelo, que podría producir la muerte o segregación temporal de especies al originarse incendios de gran magnitud. En nuestro experimento, inicialmente los disturbios no tuvieron efecto sobre la diversidad, aunque las parcelas testigo presentaron menor equitatividad por la fuerte dominancia de $P$. prionitis. Un año después, la diversidad fue mayor en las parcelas disturbadas que en las testigo, donde $P$. prionitis seguía ejerciendo una dominancia absoluta y, por lo tanto, presentaban menores valores de riqueza florística y equitatividad. Coincidentemente, Marchetti y Aceñolaza (2011) establecieron que los mayores valores medios de riqueza (S) y diversidad $\left(\mathrm{H}^{\prime}\right)$ se verificaban en pajonales que habían sido incendiados cuatro años antes.

Si bien no existieron diferencias significativas entre los tratamientos en diciembre de 2011, la riqueza fue mayor en las parcelas disturbadas. Esto podría atribuirse a que el fuego o el corte permitieron el aprovechamiento de recursos (luz y espacio) (McConnaughay and Bazzaz 1991) de los micrositios por parte de poblaciones que ya estaban al comienzo del experimento (Echinodorus grandiflorus, Chenopodiumambrosioides,Conyza bonariensis, Desmodium incanum). El corte permitió el establecimiento de Bothriochloa longipaniculata,Bromuscatharticus, Echinochloa colona, Desmanthus virgatus y Eupatorium macrocephalum. En menor medida, la quema también favoreció el ingreso de especies nuevas queno estaban desdeel inicio: Deyeuxia viridiflavescens, Hemarthriaaltissima, Vernonia glabrata var. angustifolia y Phyla nodiflora. Estos datos concuerdan con los de Fidelis et al. (2012), quienes afirman que el fuego genera micrositios que permiten el establecimiento de nuevas especies debido a la eliminación de la competencia de las matas. Feldman $y$ Lewis (2005) encontraron un incremento en la riqueza y la diversidad, incluyendo malezas, aunque la mayoría de las especies que se establecían post-disturbio eran parte del banco de semillas de la comunidad. En nuestra situación no hubo establecimiento notorio de malezas, y esto probablemente se debió a que la agricultura no está muy difundida en la región. En nuestro experimento, el fuego en el pajonal fue de severidad baja, sin cambios importantes en las temperaturas. Esto permitió el rejuvenecimiento de la especie dominante. Sin embargo, un aumento excesivo en la frecuencia de fuego puede degradar la comunidad tanto por tener impactos diferenciales sobre la composición botánica (Kunst et al. 2003) como por generar una simplificación drástica del hábitat. Inclusive, un aumento en la frecuencia del fuego podría repercutir de forma negativa sobre ciertas aves que anidan en pastizales altos; éstas podrían desaparecer si las quemas son anuales (Casillo et al. 2012).

Bajo nuestras condiciones experimentales, el tratamiento $C$ tuvo efectos positivos similares al tratamiento Q: incrementar la frecuencia de especies de mejor aptitud forrajera, probablemente por la apertura del canopeo y por el aumento de la disponibilidad de determinados nutrientes contenidos en el material cortado, que queda en el sitio. Sin embargo, si bien no hay mayores diferencias entre C y Q, es mucho más conveniente cortar que quemar, en términos de disminuir los efectos incómodos para la salud y bienestar humano y seriamente contaminantes de las quemas extensivas de pastizales del Delta y el litoral.

Agradecimientos. E.S. Massa agradece al INTA por los fondos e instalaciones para la realización de este trabajo, y al productor que permitió ubicar las parcelas experimentales en su establecimiento. L.J. Oakley, D.E. Prado y S.R. Feldman agradecen a la Universidad Nacional de Rosario haber facilitado instalaciones. D.E. Prado agradece al Consejo Nacional de Investigaciones Científicas y Técnicas (CONICET) y S.R. Feldman al Consejo de Investigaciones de la Universidad Nacional de Rosario (CIUNR). Las observaciones atinadas y constructivas de dos revisores anónimos y del cuerpo editorial de la revista contribuyeron a una mejor calidad de este artículo. 


\section{REFERENCIAS}

Aceñolaza, P., P. Zamboni, W. Sione, and F. Kalesnik. 2008. Caracterización de la región superior del complejo litoral del río Paraná: grandes unidades de ambiente. Temas de la Biodiversidad del Litoral Fluvial Argentino. INSUGEO, Misceláneas 17:293-308.

Balzarini, M. G., L. González, M. Tablada, F. Casanoves, J. P. Di Rienzo, et al. 2008. Infostat. Manual del Usuario. Editorial Brujas. Córdoba, Argentina. Pp. 336.

Bissio, J. C. 2014. Los ambientes de pajonal en el norte de Santa Fe: técnicas utilizadas para el manejo. Voces y Ecos $\mathrm{N}^{\circ}$ 32. INTA EEA Reconquista. Argentina. Pp. 65.

Braun-Blanquet, J. 1979. Fitosociología. Blume Ediciones, Madrid. España. Pp. 820.

Cabrera, A. L. 1971. Fitogeografía de la República Argentina. Boletín de la Sociedad Argentina de Botánica 14:1-42.

Casillo, J., A. Di Giacomo, and G. Marino. 2012. Quemas Controladas en pastizales. Una herramienta para el manejo de pastizales con fines productivos conservacionistas. Fundación Vida Silvestre Argentina y Aves Argentinas. Buenos Aires. Pp. 19.

Feldman, S. R., and J. P. Lewis. 2005. Effect of fire on the structure and diversity of a Spartina argentinensis tall grassland. Appl Veg Sci 8:77-84.

Feldman, S. R., J. P. Lewis, and D. Prado. 2008a. Fuego en los pajonales: ¿Desastre, negligencia o práctica ancestral? Agromensajes 25:20.

Feldman, S. R., V. Bisaro, N. Biani, and D. Prado. 2008b. Soil salinity determines the relative abundance of C3/C4 species in Argentinean grasslands. Global Ecol and Biogeography 17:308-314.

Felderhof, L., and D. Gillieson. 2006. Comparison of fire patterns and fire frequency in two tropical savanna bioregions. Austral Ecology 31:736-746.

Fidelis, A., C. Blanco, S. Müller, V. Pillar, and J. Pfadenhauer. 2012. Short-term changes caused by fire and mowing in Brazilian campos grasslands with different long-term fire histories. J Veg Sci 23:552-562.

Fidelis, A., M. D. Delgado-Cartay, C. Blanco, S. Muller, V. Pillar, et al. 2010. Fire intensity and severity in Brazilian Campos grasslands. Interciencia 35:739-745.

Figueroa Clemente, M. E., and S. Redondo Gómez. 2007. Los sumideros naturales de $\mathrm{CO}_{2}$. Muñoz Moya (eds.). Universidad de Sevilla. Sevilla. Pp. 218.

Franceschi, E. 2005. Regeneración de la vegetación herbácea luego de una inundación catastrófica en la llanura de inundación del río Paraná. Tesis Doctoral, Facultad de Ciencias Agrarias, Universidad Nacional de Rosario, Argentina. Pp. 117.

Franceschi, E., D. Prado, and J. P. Lewis. 1985. Comunidades vegetales y mapa de vegetación: Reserva "El Rico" e Islas Aledañas, Provincia de Santa Fe, República Argentina. Servicio de Publicaciones de la Universidad Nacional de Rosario. Pp. 44.

Franceschi, E., and J. P. Lewis. 1979. Notas sobre la vegetación del valle santafesino del río Paraná. Ecosur 6:55-82.

Franceschi, E., and J. P. Lewis. 1991. Early stages in the Paraná River tall grassland recovery after an extraordinary flood. Coenoses 6:47-52.

Franceschi, E., and D. Prado. 1989. Distribution of herbaceous communities of River Paraná valley along an elevation gradient after a catastrophic flood. Coenoses 4:47-53.

Heringer, I., and A. V. Jacques. 2001. Adaptação das plantas ao fogo: enfoque na transição floresta - campo. Revisão bibliográfica. Ciência Rural, Santa Maria 31:1085-1090.

Iganci, J. R. V., G. Heiden, S. T. S. Miotto, and R. T. Pennington. 2011. Campos de Cima da Serra: the Brazilian Subtropical Highland Grasslands show an unexpected level of plant endemism. Botanical Journal of the Linnean Society 167:378-393.

Jacques, A. V. 2003. A queima das pastagens naturais - efeitos sobre o solo e a vegetação. Ciência Rural, Santa Maria 33:177-181.

Kunst, C., S. Bravo, F. Moscovich, J. Herrera, J. Godoy, and S. Velez. 2003. Fecha de aplicación de fuego y diversidad de herbáceas en una sabana de Elionorus muticus (Spreng.) O. Kuntze. Revista Chilena de Historia Natural 76:105-115.

Laterra, P. 2003. Desde el Paspaletum: bases ecológicas para el manejo de pajonales húmedos con quemas prescriptas. Pp. 93-107 en: C. Kunst, S. Bravo y J. L. Panigatti (eds.). Fuego en los ecosistemas Argentinos. Ediciones INTA, Buenos Aires, Argentina. Pp. 332.

Lewis, J. P., E. A. Franceschi, and D. E. Prado. 1987. Effects of extraordinary floods on the dynamics of tall grasslands of the river Parana valley. Phytocoenologia 15:235-251.

Luisoni, L. 2006. Actividad Ganadera en el Sitio Ramsar Jaaukanigás. Pp. 91-93 en: A. R. Giraudo (ed.). Sitio Ramsar Jaaukanigás: Biodiversidad, Aspectos Socioculturales y Conservación (Río Paraná, Santa Fe, Argentina). Pp. 148.

Malvárez, A. I., and P. Kandus. 2005. El paisaje y la diversidad en las porciones superior y media del Delta del río Paraná (Argentina). Pp. 237-260 en: Humedales fluviales de América del Sur. Hacia un manejo sustentable. Universidad Nacional del Litoral/Proteger.

Marchetti, Z. Y., and P. Aceñolaza. 2005. Detección satelital y descripción de patrones de vegetación en islas del Paraná medio. Temas de las Biodiversidad del Litoral II. INSUGEO Miscelánea 14:151-158.

Marchetti, Z. Y., and P. Aceñolaza. 2011. Evaluation of the relationships between floristic heterogeneity of Panicum prionitis Ness tall grasslands and the fire history, hydrological regime and soil texture in the Paraná River floodplain, Argentina. Interciencia 36:600-607. 
McConnaughay, K. D. M., and F. A. Bazzaz. 1991. Is physical space a soil resource? Ecology 72:94-103.

McCune, B., and M. J. Mefford. 1999. PC-ORD. Multivariate analysis of ecological data. Version 4. MjM Software Design, Gleneden Beach, OR, USA. Pp. 237.

Mosconi, F. P., L. J. Priano, N. E. Hein, G. Moscatelli, and J. C. Salazar, et al. 1981. Mapa de suelos de la Provincia de Santa Fe. Tomo I. INTA - MAG Santa Fe. Pp. 248.

Neiff, J. J. 2001. Humedales de la Argentina: Sinopsis, problemas y perspectivas futuras. Centro de Ecología Aplicada (CECOAL). Corrientes, Argentina. Pp. 30.

Oakley, L. J. 1999. Botánica y sistemática de Cynodon dactylon (L.) Pers. Revista de la Facultad de Agronomía 19:3-10.

Peláez, V. D., R. Bóo, and M. D. Mayor. 2003. El fuego y la vegetación del sur del caldenal. En: C. Kunst, S. Bravo y J. L. Panigatti. (eds.). Fuego en los ecosistemas argentinos. Editorial INTA, Santiago del Estero, Argentina. Pp. 332.

Pensiero, J. F., H. F. Gutiérrez, A. M. Luchetti, E. Exner, V. Kern, et al. 2006. Flora Vascular de la provincia de Santa Fe. Claves para el reconocimiento de las familias y géneros. Catálogo sistemático de las especies. Ediciones UNL, Santa Fe. Pp. 403.

Pielou, E. C. 1969. An introduction to mathematical ecology. J. Wiley \& Sons. N. York. Pp. 286.

Ragonese, A. 1967. Vegetación y Ganadería en la República Argentina. Colección Científica del INTA. Buenos Aires. Pp. 218.

Rodrigues, C. A. G., S. M. A. Crispim, and J. A. Comastri Filho. 2002. Queima controlada no Pantanal. Corumbá EMBRAPA Pantanal, Documentos No 35. Pp. 22.

Rossi, C. A., A. De Magistris, G. González, N. Carou, and E. De Loof. 2014. Plantas de interés ganadero de la región del bajo Delta del Paraná. Ed UNLZ, Lomas de Zamora. Argentina. Pp. 206.

Sabattini, R. A., M. Wilson, N. Muzzachiodi, and A. Dorsch. 1999. Guía para la caracterización de agroecosistemas del centro-norte de Entre Ríos. Revista Científica Agropecuaria. Facultad de Ciencias Agropecuarias UNER 3:7-19.

Salvia, M., D. Ceballos, F. Grings, H. Karszenbaum, and P. Kandus. 2012. Post-fire effects in wetland environment: landscape assessment of plant coverage and soil recovery in the Paraná River Delta marshes, Argentina. Fire Ecology 8:17-37.

Secretaría de Ambiente de Entre Ríos, Centro de Desarrollo y Asistencia Tecnológica. 2016. Plan de Gestión Integrada para el Desarrollo Productivo Sostenible del Delta Entrerriano. Síntesis del informe de avance 2. Pp. 14.

Shannon, E. 1948. A mathematical theory of communication. Bell System Technology Journal 27:379-423.

Soil Taxonomy. 1999. A basic system of soil classification for making and interpreting soil surveys. 2nd ed. United States Department of Agriculture, Washington D.C. Pp. 871.

Soreng, R. J. 2010. Coleataenia Griseb. (1879): the correct name for Sorengia Zuloaga \& Morrone (2010) (Poaceae: Paniceae). Journal of the Botany Research Institute of Texas 4:691-692.

Van Der Maarel, E. 1979. Transformation of cover-abundance values in phytosociology and its effects on community similarity. Vegetatio 39:97-114. 
ES MASSA ET AL.

\section{MATERIAL SUPLEMENTARIO}

Especies presentes en el pajonal de $P$. prionitis con sus características más relevantes: familia botánica, vía fotosintética $\left(\mathrm{VF} ; \mathrm{C}_{3}, \mathrm{C}_{4}\right)$, tipo de reproducción $(\mathrm{S}=$ sexual, $\mathrm{A}=$ sexual y clonal), ciclo, hábito de crecimiento, origen $(\mathrm{N}=$ nativa, $\mathrm{E}=$ exótica), calidad forrajera ( $\mathrm{CF} ; \mathrm{MB}=$ muy buena, $\mathrm{B}=$ buena, $\mathrm{R}=$ regular, $\mathrm{m}=$ mala, $\mathrm{S} / \mathrm{I}=\sin$ información disponible).

Species recorded in the $P$. prionitis tall grassland with their relevant characteristics: botanical family, photosynthetic pathway (VF; $\mathrm{C}_{3} \mathrm{C}_{4}$ ); type of reproduction ( $\mathrm{S}=$ sexual; $\mathrm{A}=$ sexual and clonal), life cycle, growth habit; origin ( $\mathrm{N}=$ native, $\mathrm{E}=$ exotic), forage quality ( $\mathrm{CF} ; \mathrm{MB}=$ very good, $\mathrm{B}=$ good, $\mathrm{R}=$ regular, $\mathrm{M}=$ poor, $\mathrm{S} / \mathrm{I}=$ no information available)

\begin{tabular}{|c|c|c|c|c|c|c|c|}
\hline Familia & Especies & VF & $\begin{array}{l}\text { Tipo de } \\
\text { reproducción }\end{array}$ & Ciclo & $\begin{array}{l}\text { Hábito de } \\
\text { crecimiento }\end{array}$ & Origen & $\mathrm{CF}$ \\
\hline Acantaceae & Justicia leavilinguis & C3 & S & perenne & erecta & $\mathrm{N}$ & $\mathrm{m}$ \\
\hline Alismataceae & Echinodorus grandiflorus & $\mathrm{C} 3$ & S & perenne & hierba & $\mathrm{N}$ & S/I \\
\hline Amaranthaceae & Alternanthera philoxeroides & $\mathrm{C} 4$ & $\mathrm{~A}$ & perenne & postrada & $\mathrm{N}$ & $\mathrm{B}$ \\
\hline Apiaceae & Eryngium ebracteatum & $\mathrm{C} 3$ & $S$ & perenne & roseta & $\mathrm{N}$ & $\mathrm{m}$ \\
\hline Araliaceae & Hydrocotyle bonariensis & $\mathrm{C} 3$ & $\mathrm{~A}$ & perenne & palustre & $\mathrm{N}$ & $\mathrm{R}$ \\
\hline Asteraceae & Acmella decumbens & $\mathrm{C} 3$ & A & perenne & erecta & $\mathrm{N}$ & $\mathrm{m}$ \\
\hline Asteraceae & Aspilia montevidensis & $\mathrm{C} 3$ & $S$ & perenne & erecta & $\mathrm{N}$ & $\mathrm{m}$ \\
\hline Asteraceae & Baccharis trimera & $\mathrm{C} 3$ & S & perenne & subarbusto & $\mathrm{N}$ & $\mathrm{m}$ \\
\hline Asteraceae & Conyza bonariensis & C3 & S & anual & roseta & $\mathrm{N}$ & $\mathrm{m}$ \\
\hline Asteraceae & Eupatorium arnottianum & C3 & S & perenne & erecta & $\mathrm{N}$ & $\mathrm{m}$ \\
\hline Asteraceae & Eupatorium lanigerum & $\mathrm{C} 3$ & $S$ & perenne & erecta & $\mathrm{N}$ & $\mathrm{m}$ \\
\hline Asteraceae & Eupatorium macrocephalum & $\mathrm{C} 3$ & S & perenne & erecta & $\mathrm{N}$ & $\mathrm{m}$ \\
\hline Asteraceae & Gamochaeta coarctata & $\mathrm{C} 3$ & S & perenne & roseta & $\mathrm{N}$ & $\mathrm{m}$ \\
\hline Asteraceae & Mikania cordifolia & $\mathrm{C} 3$ & $S$ & perenne & voluble & $\mathrm{N}$ & $\mathrm{m}$ \\
\hline Asteraceae & Mikania periplocifolia & $\mathrm{C} 3$ & S & perenne & voluble & $\mathrm{N}$ & $\mathrm{m}$ \\
\hline Asteraceae & Vernonia glabrata var. angustifolia & C3 & $\mathrm{A}$ & perenne & erecta & $\mathrm{N}$ & $\mathrm{m}$ \\
\hline Asteraceae & Xanthium spinosum & $\mathrm{C} 3$ & $S$ & anual & erecta & $\mathrm{E}$ & $\mathrm{m}$ \\
\hline Cariophyllaceae & Stellaria media & $\mathrm{C} 3$ & $S$ & anual & hierba & $\mathrm{E}$ & $\mathrm{R}$ \\
\hline Chenopodiaceae & Chenopodium ambrosioides & $\mathrm{C} 3$ & $\mathrm{~S}$ & perenne & erecta & $\mathrm{N}$ & $\mathrm{m}$ \\
\hline Cyperaceae & Cyperus entrerianus & $\mathrm{C} 4$ & $\mathrm{~A}$ & perenne & rizomatosa & $\mathrm{N}$ & $\mathrm{m}$ \\
\hline Cyperaceae & Cyperus sp. & $\mathrm{C} 4$ & $\mathrm{~A}$ & perenne & rizomatosa & $\mathrm{N}$ & $\mathrm{m}$ \\
\hline Cyperaceae & Eleocharis elegans & C4 & $\mathrm{A}$ & perenne & rizomatosa & $\mathrm{N}$ & $\mathrm{R}$ \\
\hline Cyperaceae & Eleocharis nodulosa & $\mathrm{C} 4$ & A & perenne & rizomatosa & $\mathrm{N}$ & $\mathrm{R}$ \\
\hline Cyperaceae & Fimbristylis squarrosa & $\mathrm{C} 4$ & A & perenne & hierba & $\mathrm{N}$ & $\mathrm{R}$ \\
\hline Cyperaceae & Rhynchospora corymbosa & $\mathrm{C} 3$ & $\mathrm{~A}$ & perenne & hierba & $\mathrm{N}$ & $\mathrm{m}$ \\
\hline Cyperaceae & Schoenoplectus californicus & $\mathrm{C} 3$ & $\mathrm{C}$ & perenne & hierba & $\mathrm{N}$ & $\mathrm{m}$ \\
\hline Convolvulaceae & Convolvulus arvensis & $\mathrm{C} 3$ & $\mathrm{~S}$ & perenne & rastrera & $\mathrm{E}$ & $\mathrm{m}$ \\
\hline Fabaceae & Desmanthus virgatus & C3 & S & perenne & rastrera & $\mathrm{N}$ & B \\
\hline Fabaceae & Desmodium incanum & C3 & S & perenne & semi erecta & $\mathrm{N}$ & $\mathrm{MB}$ \\
\hline Fabaceae & Dolichopsis paraguariensis & $\mathrm{C} 3$ & $S$ & perenne & postrada & $\mathrm{N}$ & MB \\
\hline Fabaceae & Mimosa strigillosa & $\mathrm{C} 3$ & $S$ & perenne & postrada & $\mathrm{N}$ & $\mathrm{MB}$ \\
\hline Iridaceae & Sisyrinchium sp. & $\mathrm{C} 3$ & $\mathrm{~A}$ & perenne & erecta & $\mathrm{N}$ & $\mathrm{m}$ \\
\hline Lamiaceae & Hyptis lappacea & $\mathrm{C} 3$ & $S$ & perenne & erecta & $\mathrm{N}$ & $\mathrm{m}$ \\
\hline Lamiaceae & Lamium amplexicaule & $\mathrm{C} 3$ & $S$ & perenne & erecta & $\mathrm{E}$ & $\mathrm{m}$ \\
\hline Lythraceae & Cuphea glutinosa & $\mathrm{C} 3$ & S & perenne & hierba & $\mathrm{N}$ & $\mathrm{m}$ \\
\hline Malvaceae & Sida rhombifolia & C3 & $S$ & perenne & erecta & $\mathrm{N}$ & $\mathrm{m}$ \\
\hline Onagraceae & Ludwigia peploides & $\mathrm{C} 3$ & S & perenne & palustre & $\mathrm{N}$ & $\mathrm{B}$ \\
\hline Oxalidaceae & Oxalis corniculata & $\mathrm{C} 3$ & S & perenne & postrada & $\mathrm{N}$ & $\mathrm{m}$ \\
\hline Poaceae & Acroceras zizanioides & $\mathrm{C} 4$ & $S$ & perenne & rastrera & $\mathrm{N}$ & $\mathrm{B}$ \\
\hline Poaceae & Bothriochloa longipaniculata & $\mathrm{C} 4$ & S & perenne & cespitosa & $\mathrm{N}$ & $\mathrm{B}$ \\
\hline Poaceae & Bromus catharticus & $\mathrm{C} 3$ & S & perenne & cespitosa & $\mathrm{N}$ & $\mathrm{MB}$ \\
\hline Poaceae & Chascolytrum subaristatum & $\mathrm{C} 3$ & $\mathrm{~A}$ & perenne & cespitosa & $\mathrm{N}$ & $\mathrm{MB}$ \\
\hline Poaceae & Chascolytrum uniolae & $\mathrm{C} 3$ & $\mathrm{~A}$ & perenne & mata & $\mathrm{N}$ & $\mathrm{R}$ \\
\hline Poaceae & Cynodon dactylon & $\mathrm{C} 4$ & A & perenne & rastrera & $\mathrm{E}$ & $\mathrm{R}$ \\
\hline Poaceae & Deyeuxia viridiflavescens & C3 & $\mathrm{A}$ & perenne & cespitosa & $\mathrm{N}$ & $\mathrm{B}$ \\
\hline Poaceae & Echinochloa colona & $\mathrm{C} 4$ & $\mathrm{~A}$ & anual & cespitosa & $\mathrm{N}$ & $\mathrm{B}$ \\
\hline Poaceae & Eragrostis lugens & $\mathrm{C} 4$ & S & perenne & cespitosa & $\mathrm{N}$ & $\mathrm{R}$ \\
\hline Poaceae & Hemarthria altissima & $\mathrm{C} 4$ & $\mathrm{~A}$ & perenne & cespitosa & $\mathrm{E}$ & $\mathrm{B}$ \\
\hline Poaceae & Leersia hexandra & $\mathrm{C} 3$ & A & perenne & cespitosa & $\mathrm{N}$ & $\mathrm{B}$ \\
\hline Poaceae & Panicum prionitis & $\mathrm{C} 4$ & A & perenne & mata & $\mathrm{N}$ & $\mathrm{m}$ \\
\hline Poaceae & Paspalum intermedium & $\mathrm{C} 4$ & A & perenne & mata & $\mathrm{N}$ & $\mathrm{R}$ \\
\hline Poaceae & Paspalum plicatulum & $\mathrm{C} 4$ & $\mathrm{~A}$ & perenne & cespitosa & $\mathrm{N}$ & $\mathrm{B}$ \\
\hline Poaceae & Paspalum urvillei & $\mathrm{C} 4$ & $\mathrm{~A}$ & perenne & cespitosa & $\mathrm{N}$ & $\mathrm{B}$ \\
\hline Poaceae & Phalaris angusta & $\mathrm{C} 3$ & $S$ & anual & cespitosa & $\mathrm{N}$ & $\mathrm{MB}$ \\
\hline Poaceae & Poa annua & C3 & S & anual & cespitosa & $\mathrm{E}$ & $\mathrm{MB}$ \\
\hline Poaceae & Sacciolepis vilvoides & $\mathrm{C} 4$ & $\mathrm{~A}$ & perenne & rastrera & $\mathrm{N}$ & $\mathrm{B}$ \\
\hline Poaceae & Setaria parviflora & $\mathrm{C} 4$ & S & perenne & cespitosa & $\mathrm{N}$ & $\mathrm{R}$ \\
\hline Polygonaceae & Polygonum acuminatum & C3 & $S$ & perenne & postrada & $\mathrm{N}$ & $\mathrm{m}$ \\
\hline Polygonaceae & Polygonum convulvulus & $\mathrm{C} 3$ & $S$ & anual & postrada & $\mathrm{E}$ & $\mathrm{m}$ \\
\hline Pteridaceae & Pteris tremula & $\mathrm{C} 3$ & $S$ & perenne & helecho & $\mathrm{E}$ & $\mathrm{m}$ \\
\hline Solanaceae & Nierembergia aristata & $\mathrm{C} 3$ & $S$ & perenne & erecta & $\mathrm{E}$ & $\mathrm{m}$ \\
\hline Solanaceae & Solanum glaucophyllum & $\mathrm{C} 3$ & $\mathrm{~A}$ & perenne & arbusto & $\mathrm{N}$ & $\mathrm{m}$ \\
\hline Urticaceae & Parietaria debilis & $\mathrm{C} 3$ & $S$ & anual & rastrera & $\mathrm{E}$ & $\mathrm{m}$ \\
\hline Verbenaceae & Phyla nodiflora & C3 & S & perenne & rastrera & $\mathrm{N}$ & $\mathrm{m}$ \\
\hline
\end{tabular}

